\title{
Field-induced ordering phenomena and non-local elastic compliance in two-dimensional colloidal crystals
}

\author{
K Franzrahe ${ }^{1}$, P Nielaba ${ }^{1}$, A Ricci ${ }^{2}$, K Binder ${ }^{2}$, S Sengupta ${ }^{3}$, \\ P Keim ${ }^{1}$ and G Maret ${ }^{1}$ \\ ${ }^{1}$ Department of Physics, University of Konstanz, Universitätstraße 10, \\ D-78457 Konstanz, Germany \\ 2 Department of Physics, Johannes Gutenberg-University, Staudinger Weg 7, \\ D-55099 Mainz, Germany \\ ${ }^{3}$ Satyendra Nath Bose National Centre for Basic Sciences, Block-JD, Sector-III, Salt Lake, \\ Kolkata 700 098, India \\ E-mail: Kerstin.Franzrahe@uni-konstanz.de
}

\begin{abstract}
Ordering phenomena in colloidal dispersions exposed to external one-dimensional, periodic fields or under confinement are studied systematically by Monte Carlo computer simulations. Such systems are useful models for the study of monolayers on a substrate. We find that the interaction with a substrate potential completely changes the miscibility of a binary, hard disc mixture at low external field amplitudes. The underlying ordering mechanisms leading to this laser-induced de-mixing differ, depending on which components interact with the substrate potential. Generic effects of confinement on crystalline order in two dimensions are studied in a model system of point particles interacting via a potential $\propto r^{-12}$. The state of the system (a strip of width $D$ ) depends very sensitively on the precise boundary conditions at the two confining walls. Commensurate, corrugated boundary conditions enhance both orientational order and positional order. In contrast, smooth repulsive boundaries enhance only the orientational order and destroy positional (quasi-)long range order. As external fields have a strong impact on the elastic behaviour of colloidal crystals there is a need to analyse the elastic response in such systems for the field-free case first. To this aim we study the strain-strain correlation functions in a two-dimensional crystal formed by super-paramagnetic colloids, as monitored by standard video microscopy.
\end{abstract}

Minimization trends in physics and technology have caused a lot of interest lately in monolayers and their interactions with a substrate. The assembly of nanoparticles into spatially extended regular structures is the first step towards a new generation of materials and devices. Colloidal dispersions in external fields or under confinement are valuable model systems for the systematic study of such settings, as they are experimentally directly accessible via laser scanning microscopy. In addition their effective interactions can be tailored to specific needs by various means. In two dimensions (2d) such systems are often used for the study of monolayers on a substrate. Monodisperse $2 \mathrm{~d}$ colloidal systems with a substrate potential have been studied extensively in experiments [1, 2], computer simulations [3-8] and theory [9-11] over the last few decades. Re-entrant phase transition scenarios like laser-induced freezing (LIF) and laser-induced melting (LIM) have been observed in such settings. We address the question of how the addition of another length scale to such a system influences the intricate competition between adsorbate-adsorbate interaction and adsorbate-substrate interaction by studying a binary $50 \%$ mixture under the influence of a one-dimensional (1d) spatially 
periodic substrate potential in section 1. Another way to influence the order in $2 \mathrm{~d}$ systems is by geometric confinement, which will be important in the development of novel devices. Depending on the precise settings confinement can either enhance or destroy ordered structures. In section 2 we deal with the generic effects of geometric confinement by analysing a system of model colloids confined within a quasione-dimensional strip between two parallel boundaries using Monte Carlo simulations. External fields not only influence the ordering, but also the elastic properties of the colloidal dispersions. These are also an important aspect in the search for novel material in soft matter physics. In order to gain a deeper understanding of the elastic response in such systems, we study the strain-strain correlation functions of a monodisperse, $2 \mathrm{~d}$, triangular colloidal crystal in section 3 .

\section{A binary mixture in a $1 d$ spatially periodic potential}

The intricate competition between adsorbate-adsorbate and adsorbate-substrate interactions is the origin of a complex phase behaviour observed in monodisperse monolayers on a substrate. Re-entrant phase transitions like LIF and LIM are known to occur in various systems (e.g. [2, 4, 5]). In this section we show that adding another competing length scale to such a set-up generates novel interesting phenomena [12-14]. We will concentrate in this section on a regime with low external potential amplitudes $V_{0} /\left(k_{\mathrm{B}} T\right) \leqslant 1.5$. Following the approaches in the studies of monodisperse systems, we use an external potential given by

$$
V(\vec{r})=V_{0} \sin (\vec{K} \cdot \vec{r}), \quad \vec{K}={ }_{a}^{4 \pi}(1,0) .
$$

Here $a$ is the lattice parameter of the $S_{1}(A B)$ lattice [15], which provides the densest packing for the studied equimolar binary mixture (diameter ratio $\sigma_{B} / \sigma_{A}=0.414$ ). All lengths are measured in units of $\sigma_{A}$, therefore the dimensionless number density is $\varrho={ }_{A}^{N} \sigma_{A}^{2}$. A wavelength $\lambda=2 \pi /|\vec{K}|$, commensurate to the lattice planes of this crystalline structure, is chosen for the periodicity of the substrate potential. The situation is schematically illustrated in figure 1 . The colloidal particles are modelled by hard discs. Monte Carlo simulations in the $N V T$ ensemble with periodic boundary conditions are employed. In order to facilitate equilibration we use a cluster move [16] and additional non-local moves, in which particle displacements, which are integer multiples of the potential wavelength, are attempted.

Even in the field-free case binary mixtures in $2 \mathrm{~d}$ are known to show interesting phenomena. As has been recently observed experimentally [17], introducing small particles into a system of large particles breaks the spanning network of large particles. This results in a competition between free volume and configurational entropy and leads to clustering [18] and structural crossover $[17,19]$. Nevertheless in a purely repulsive system the effect is too weak to drive phase separation. In general one needs to distinguish the following three cases:

(a) only the smaller component interacts with the external field;

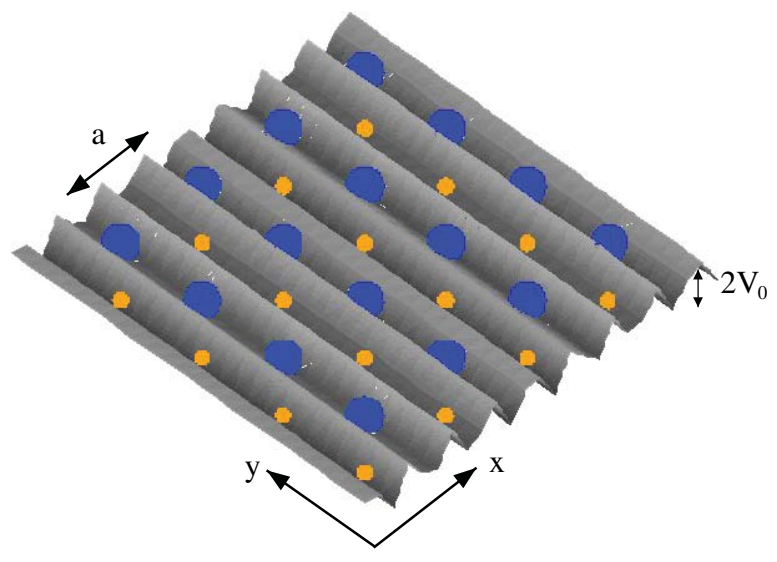

Figure 1. Schematic illustration of the external, 1d spatially periodic substrate potential $V(\vec{r})$ and the $S_{1}(A B)$ lattice structure relative to it. The larger component of the binary mixture is displayed in black (online: blue), the smaller in grey (online: orange). $a$ is the lattice parameter of the $S_{1}(A B)$ lattice, while $V_{0}$ is the amplitude of the external potential.

(b) both components interact with the external field;

(c) only the larger component interacts with the external field.

For a detailed discussion of the phase behaviour for case $(a)$, when only the smaller component interacts with the external potential, see [12]. In summary we find for this case that at intermediate to high external field amplitudes the coupling to the external field induces a coexistence of a $S_{1}(A B)$ crystal with an equimolar fluid. This regime is followed by a fissuring regime for higher dimensionless number densities $\varrho$, in which the larger component forms a square lattice. This lattice structure has a tendency to form fissures parallel to the modulated direction of the external field, in which the smaller components can accumulate.

For low potential amplitudes $V_{0}$ we find a novel phenomenon. In contrast to the monodisperse LIF scenario a laser-induced de-mixing sets in for all three cases. We observe a coexistence of a small component enriched fluid with a droplet of a monodisperse crystalline structure formed by the larger component. This is visualized in figure 2, where overlays of all configurations of the simulation (corrected for the centre-of-mass movement) are shown. The positions of the larger component are denoted by black (online: blue) points, while those of the smaller component are plotted in grey (online: orange). The data was taken at a number density $\varrho=1.71$. A heuristic argument by Buhot et al [20] yields a diameter ratio of $\sigma_{B} / \sigma_{A}=1 / 100$ as the upper limit for possible phase separation in binary hard disc mixtures. Nevertheless, exposing an equimolar binary mixture with diameter ratio $\sigma_{B} / \sigma_{A}=0.414$ to an external field interferes with the competition of free volume and configurational entropy in a controlled way by introducing the constraint of energy minimization. Phase separation is induced. Subfigure 2(c) shows case (c) at $V_{0} / k_{\mathrm{B}} T=0.5$. The formation of the monodisperse lattice structure is induced directly by the interaction with the external field in this case. The resulting structure is aligned in its orientation with the minima of the potential. As the periodicity of the 

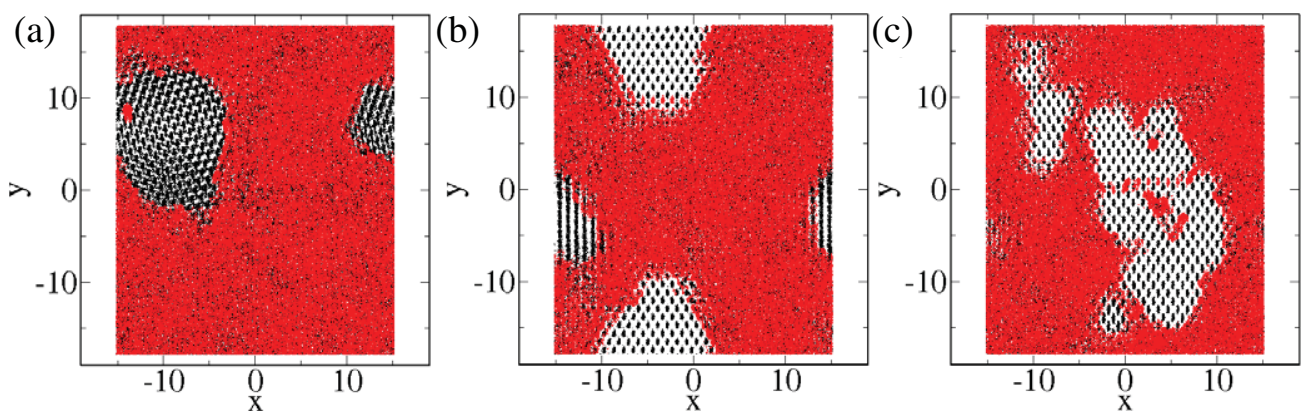

Figure 2. The laser-induced de-mixing regime. Shown are overlays of centre-of-mass motion corrected configurations from simulations at $\varrho=1.71$. The positions of the larger component are displayed in black, those of the smaller component in grey (online: red). (a) $V_{0} / k_{\mathrm{B}} T=0.6$ for case (a), (b) $V_{0} / k_{\mathrm{B}} T=0.3$ for case (b) and (c) $V_{0} / k_{\mathrm{B}} T=0.5$ for case $(c)$.

external potential is not commensurate with a monodisperse triangular lattice of the larger component, a rhombic structure forms. In contrast at $V_{0} / k_{\mathrm{B}} T=0.6$ the monodisperse crystalline structure observed for case $(a)$, when only the smaller component interacts with the external field, is a triangular lattice. This difference can be explained by the ordering mechanism, which leads to the de-mixing in this case. As the smaller component tries to optimize its alignment with the potential minima it forms chains along the $y$ direction. This chain formation has a lower number density than the fluid mixture. Under the constraint of a fixed overall number density, the larger component is thus indirectly induced to form a dense packing, i.e. a triangular lattice structure in order to facilitate the energy minimization of the smaller components. For case (b), when both components interact with the substrate potential, both types of ordering mechanisms are at work. Therefore only at even lower potential amplitudes do we observe a pure de-mixing, as shown in subfigure 2(b). The de-mixing is followed by a regime in which the competition of the ordering mechanisms leads to a domain growth of rhombic monodisperse and square lattice bi-disperse areas.

In conclusion, we have shown via Monte Carlo simulations that the miscibility of a binary hard disc mixture can be tuned in a controlled way by exposing the mixture to a one-dimensional spatially periodic potential. Weak external fields induce a phase separation into an ordered monodisperse phase of the larger component and a disordered fluid phase.

\section{Confinement: a quasi-one-dimensional system}

In this section we analyse the nature of the ordered phase for a model of colloidal particles confined within a quasi-onedimensional (q-1d) strip between two parallel boundaries, or walls, separated a distance $D$ in two dimensions $(2 \mathrm{~d})^{4}$. Using Monte Carlo simulations we find $[22,23]$ that at densities typical of the bulk $2 \mathrm{~d}$ triangular solid the order in the q-1d strip is determined by the nature of the boundaries. While for a suitably corrugated boundary potential order is enhanced, for a uniformly repulsive smooth boundary potential only ordering normal to the walls is enhanced ('layering'); parallel to the wall it is destroyed.

\footnotetext{
${ }^{4}$ For colloidal crystals in $d=3$ confined in narrow slit pores, see [21].
}

Ordering in strips and half-planes have been studied $[24-28,21,29]$ in the past for spin systems primarily in the context of surface critical phenomena where both suppression or enhancement of order near free boundaries have been observed.

Studies of the nature of ordering for quasi-1d strips have been done for Wigner crystals [30], repulsive magnetorheological (MR) colloids [31, 32] and for colloids between walls in non-equilibrium situations [33], for instance. The former system is relevant, for example, for electrons on the surface of liquid helium that is confined in a quasi-1d channel, where unusual ordering phenomena were found [34], and for confined dusty plasmas [35]. MR colloids under confinement are of interest for microfluidic applications, see [31,32] for references and discussions of further related systems. Unusual mechanical behaviour of a related system has also been reported [36], and in [31] it was noted that the properties of the confined system approach those of the unbounded system surprisingly slowly as the channel width is increased. Early experiments on a model hard disc system [37] also showed unconventional behaviour. Clearly, the understanding of confined $2 \mathrm{~d}$ crystals is far from being complete, and it is the purpose of the present work to contribute to a resolution of these puzzles by combining simulations of a generic model with a suitable theoretical analysis.

For finite $D$, the system is quasi-one-dimensional, and hence one might argue that long range order (LRO) is destroyed, for systems with short range forces [24]. Indeed, a 1d harmonic crystal displays a fluid-like structure factor [38] and this accounts well for real quasi-1d chain compounds such as $\mathrm{Hg}_{3-\delta} \mathrm{AsF}_{6}$ (where $\delta \ll 1$ ) [39]. However, for confined colloidal crystals the situation should be more subtle, since the confinement between hard repulsive boundaries reduces fluctuations in the direction normal to the boundaries, causing a pronounced 'layering effect'. Ordering is also strongly influenced by the boundaries, for example it is known that a boundary corrugation potential $[24,40]$ enhances the order parallel to the boundaries.

The interaction among colloidal particles may be conveniently parametrized by $V(r)=\varepsilon(\sigma / r)^{n}$ where the exponent may vary from $n=\infty$ in the case of hard sphere colloids [41-44] to $n=3$ for super-paramagnetic [45, 46] dipolar colloids. We have chosen a potential with $n=12$ 
and with a cutoff at $r_{\mathrm{c}}=5 \sigma$, at a temperature $k_{\mathrm{B}} T / \varepsilon=1$ (where $k_{\mathrm{B}}$ is the Boltzmann constant) and density (choosing units such that $\sigma=1) \varrho=1.05$. This choice retains the advantage of a smooth potential which is also sufficiently short ranged for computational convenience. Further, we believe that the phenomena reported here are independent of the detailed nature of the potential. At the chosen density melting in the bulk occurs for $k_{\mathrm{B}} T / \varepsilon \approx 1.35$ in this model [47]. We choose two types of wall potentials, with walls oriented parallel to lattice axes of the triangular lattice: (i) (planar walls) $V_{\text {wall }}(\mathbf{r})=\varepsilon_{\text {wall }}\left(\sigma /\left|x-x_{\text {wall }}\right|\right)^{10}$ for a particle at position $\mathbf{r}$, where the $x$ direction is chosen perpendicular to the boundaries, and the positions $x_{\text {wall }}$ of the two walls are a distance $D$ apart that is an integer multiple of the distance $a \sqrt{3} / 2, a$ being the lattice spacing of an ideal triangular lattice compatible with the chosen density. The strength of this potential is typically $\varepsilon_{\text {wall }}=0.0005 k_{\mathrm{B}} T$ (also much stronger wall potentials were tested). (ii) Structured walls causing a periodic corrugation of the potential were created by choosing two rows of particles fixed in the positions of this ideal triangular lattice, and these particles interact with the mobile particles with the same potential $V(\mathbf{r})$ as specified above. Summing up these potentials due to the fixed particles defines the corrugation potential $V_{\text {struc }}(\mathbf{r})$ (structured walls) of such structured boundaries. A sketch of the configurations in front of the two wall types is given in figure 3.

We use standard Monte Carlo methods [48], where single particles are selected at random to attempt a small random displacement in a square of linear dimension $\kappa=0.206$ centred at the old position of the particle. Typical runs were performed for systems containing between $D \times L=20 \times 20$ and $D \times L=60 \times 60$ particles, carrying out $10^{6}$ Monte Carlo steps (MCS) per particle in each run. In the direction parallel to the walls, periodic boundary conditions are used. For the sake of comparison, runs for 'bulk' systems (with no walls and periodic boundary conditions in both directions) were also done. Some runs were also made for a strongly elongated geometry $(D \times L=20 \times 500)$ to probe the behaviour of displacement correlations at large length scales.

Both types of boundaries enhance the order in the $x$ direction near the walls. This enhancement of order in the direction normal to the walls is even more pronounced (and of larger range) when we approach the transition to the fluid phase $[22,23]$. While in the bulk the well-defined orientational LRO and nonzero shear modulus of the $2 \mathrm{~d}$ crystal have both disappeared in the fluid, some wall-induced orientational LRO persists in the thin strip [22, 23].

In contrast, in the direction parallel to the boundaries the behaviour in the two cases differs dramatically: for the structured boundary, the structure factor $S(q)$ exhibits the sharp Bragg peaks expected for a crystal ${ }^{5}$ (figure 4(a)). For the structureless repulsive boundary, a typical fluid-like structure factor results, which is almost in quantitative agreement with

\footnotetext{
5 Note that for orderings commensurate with the corrugation potential the statement of Mermin [49] that crystals in $d=2$ have only orientational LRO and no positional LRO does not apply here. This situation is reminiscent of the behaviour of thin films adsorbed on substrates where true LRO commensurate with the substrate corrugation potential can occur [40].
}

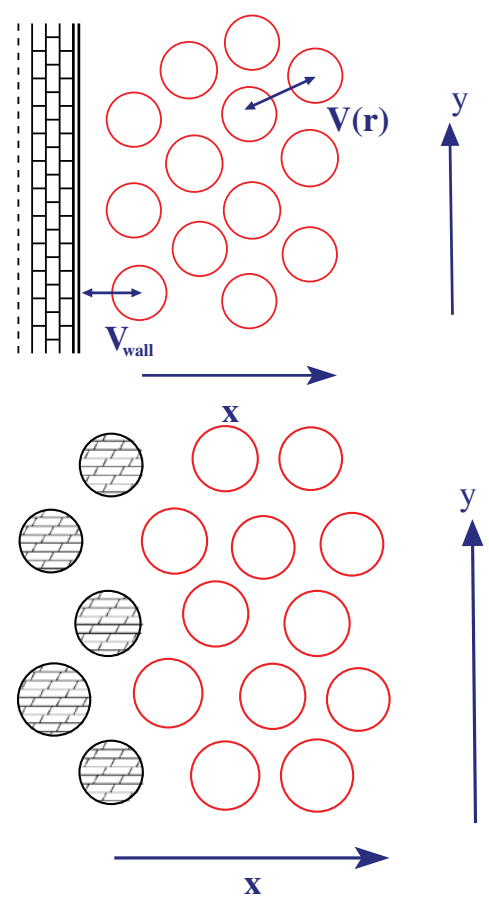

Figure 3. Schematic descriptions of the two types of walls used in this paper in order to confine the triangular crystal. Walls are always oriented along the $y$ axis. The upper part shows the case of planar walls, described by a repulsive potential $V_{\text {wall }}^{\text {planar }}=\epsilon_{\text {wall }}(\sigma / \mid x-$ $\left.x_{\text {wall }} \mid\right)^{10}$. The position $x_{\text {wall }}$ is chosen such that it replaces a row of particles otherwise present at this distance. The lower part shows the case of 'structured walls', created by fixing two rows of particles precisely at the positions of the ideal triangular lattice (with the same lattice spacing $a_{0}$ as in the bulk). The frozen particles interact with the mobile ones with the same pair potential $V(r)$ as is acting between two mobile particles a distance $r$ apart. In this way, 'structured' walls provide a potential corrugated in the $y$ direction and precisely commensurate with the considered crystal structure.

a fit to the $S(q)$ for 1d harmonic chains [38] (figure 4(b)), although we deal here with a system of 30 rows confined between two boundaries, rather than a true $1 \mathrm{~d}$ system. This reduction of order in the $y$ direction along the boundaries is also seen directly when one superimposes the positions from 1000 configurations of the particles (figure 4 inset). This lack of order concerns the positional LRO only, however: the orientational LRO due to the strong confinement in layers parallel to the walls is rather well developed, and even better for the planar walls rather than the structured walls $[22,23]$. The analogy to $1 \mathrm{~d}$ harmonic chains suggests that for $T \rightarrow 0$ the range of positional LRO gets gradually very large, but true LRO only occurs for $T=0$. (Note that also Ising model strips of finite width have a transition only at $T=0$ for any finite value of $D$, but there the correlation length below the critical temperature $T_{\mathrm{c}}$ of the $2 \mathrm{~d}$ model is much larger, of the order $D \exp ($ constant $\times D)$.)

The fact that confinement by planar structureless boundaries turns a colloidal $2 \mathrm{~d}$ crystal into a kind of smectic phase [50] (or strongly modulated fluid, respectively) also shows up when one examines 'bulk' properties of the strip like the elastic constants (figure 5). While for structured walls one reaches the behaviour of the bulk rapidly, for planar walls one 

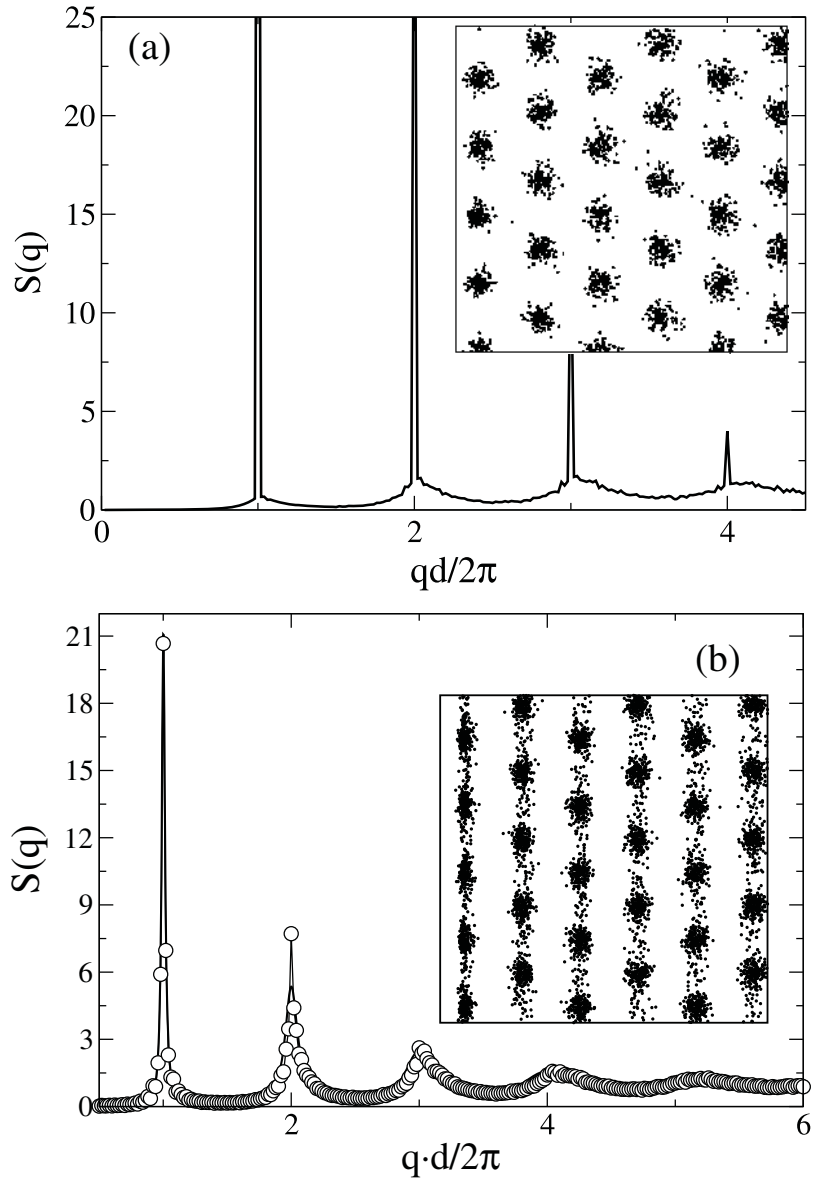

Figure 4. Static structure factor $S(q)$ plotted versus $q d / 2 \pi$ ( $d$ being the distance between lattice axes in the $y$ direction parallel to the boundaries), for structured walls (a) and planar walls (b). All data are for systems of 900 particles. In case (b) a fit to the $S(q)$ for a harmonic chain [38] is included. The wavevector $\mathbf{q}$ is oriented along the $y$ direction. The insets in (a) and (b) show the corresponding particle configurations for eight layers adjacent to the wall on the left; 1000 configurations out of a run lasting $10^{6} \mathrm{MCS}$ are superimposed after putting the centre of mass into the origin of the coordinate system each time. Note the prominent anisotropy of the density peaks in the inset of (b).

rather has $C_{33}$ (bulk) $\sim 2 C_{33}(n)$, which implies a vanishing shear modulus. The elastic constants could be determined directly from an analysis of the configurations of the particles applying the method of Sengupta et al [51, 52]. Particularly remarkably the increase of strip thickness (or number $n$ of rows in the strip, respectively) does not cause any visible approach to bulk behaviour. The question how, for the elastic constants, the thermodynamic limit is approached is intriguing. The planar boundary provides an elastic distortion of long range [53] to the crystal, and our results imply that in $d=2$ this distortion disturbs the positional LRO.

This lack of positional LRO may be understood from a calculation of the displacement correlation function $B(y)=$ $\left\langle\left[u_{y}(y)-u_{y}(o)\right]^{2}\right\rangle$, where $u_{y}$ is the displacement away from a reference lattice in the direction parallel to the walls and the angular brackets signify ensemble averaging. Using a harmonic elastic Hamiltonian for the strip geometry, this may
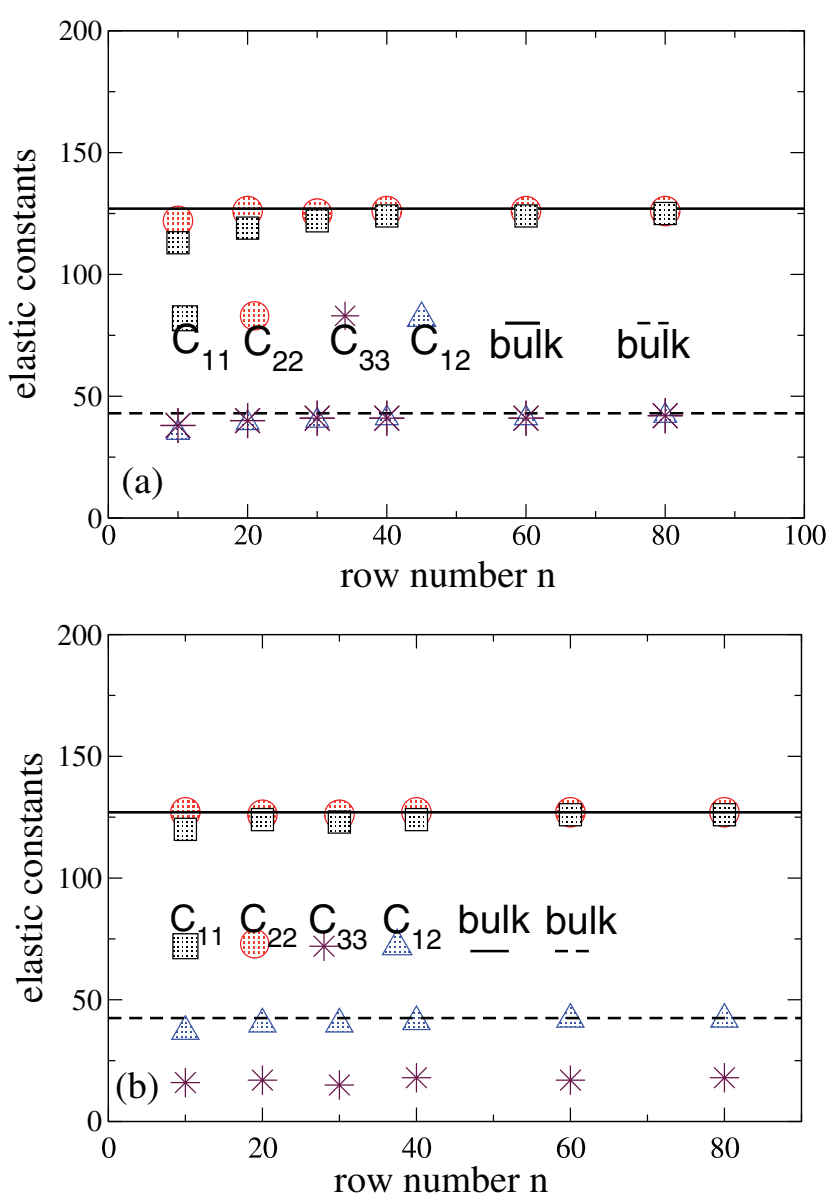

Figure 5. Elastic constants in units of $k_{\mathrm{B}} T / \sigma^{2}$ for structured walls (a) and planar walls (b) plotted versus the number of rows $n$ between the walls. The Voigt notation for the elastic constants is used. Horizontal straight lines show the bulk values of the corresponding triangular crystal, for which the symmetries $C_{11}=C_{22}$ and $C_{12}=C_{33}$ hold.

$$
\begin{aligned}
& \text { be written as } \\
& \qquad B(y)=2 \sum_{\substack{q_{x}={ }^{2 \pi n_{x}} \\
D}} \sum_{q_{y}={ }_{L}^{2 \pi n_{y}}}\left\langle\left|u_{\mathbf{q}}^{2}\right|\right\rangle\left[1-\cos \left(q_{y} y\right)\right] \\
& \left\langle\left|u_{\mathbf{q}}^{2}\right|\right\rangle=\underset{(\lambda+2 \mu-P) q^{2}}{k_{\mathrm{B}} T} \hat{q}_{y}^{2}+\underset{(\mu-P) q^{2}}{k_{\mathrm{B}} T}\left(1-\hat{q}_{y}^{2}\right) .
\end{aligned}
$$

Here, $n_{x}$ and $n_{y}$ are integers, $\hat{q}_{y}=q_{y} / q$ and the Lamé coefficients $\lambda$ and $\mu$ are those of a bulk soft disc solid at the same density, while $P$ is the overall (nonzero) hydrostatic pressure [51, 52]. The result of this calculation is shown in figures 6(a) and (b). We observe a crossover from the logarithmic increase of mean-square displacements $B(y) \propto \ln y$ with distance $y$ (characteristic for $2 \mathrm{~d}$ solids) to a linear increase (1d systems [38]) at a distance of order $D \ln (D / a)$ [22, 23] which is cut off by the periodic boundary conditions for small systems. The direct evaluation of $B(y)$ for the soft disc system with periodic boundary conditions is also shown in figure 6(a) and agrees well with the harmonic calculation within the error bars of the former. The corresponding calculation with planar walls shows an enhancement of the displacement fluctuations parallel to the walls for the lattice layer closest to the wall figure 6(b). 

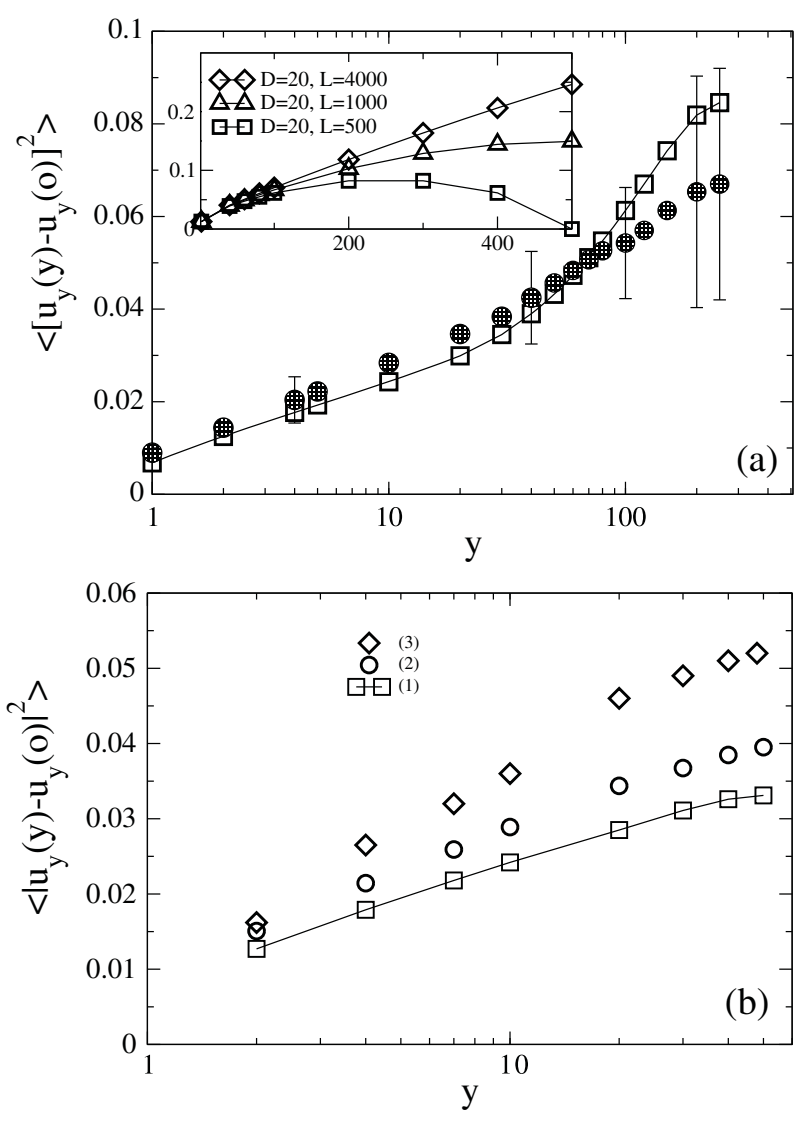

Figure 6. (a) Comparison of $\left\langle\left[u_{y}(y)-u_{y}(o)\right]^{2}\right\rangle$ versus $y$ (in logarithmic scale) for the soft disc solid at $\rho \sigma^{2}=1.05$ and $k_{\mathrm{B}} T / \epsilon=1$ (filled circles) with the results of the harmonic theory ( $\square$ ) for a system with $D=20$ and $L=500$. The input Lamé coefficients $\lambda=42$ and $\mu=41$ in units of $k_{\mathrm{B}} T / \sigma^{2}$ are those of the bulk soft discs at the same $\rho$ and $T$, with the pressure $P=17.4$ in units of $\rho k_{\mathrm{B}} T$. Inset shows the crossover of $\left\langle\left[u_{y}(y)-u_{y}(o)\right]^{2}\right\rangle$ in the harmonic approximation from an initial logarithmic to a linear growth for various $D$ and $L$ signifying destruction of positional LRO. Note that for small systems the linear part is cut off due to finite size. (b) Comparison of $\left\langle\left[u_{y}(y)-u_{y}(o)\right]^{2}\right\rangle$ versus $y$, for the harmonic solid (1) (the line is a guide to the eye), soft disc solid with periodic boundary conditions (2) and soft disc solid with planar walls (3) at $\rho \sigma^{2}=1.05$ and $k_{\mathrm{B}} T / \epsilon=1$. All three systems have 2000 particles $(D=20$ and $L=100)$. The displacement correlations are calculated only for the layer closest to the wall for (3). Note the enhancement of fluctuations for the system with planar walls.

This behaviour is quite similar to that seen in the XY model [29] with free boundary conditions. Fluctuations of the displacement perpendicular to the walls are, of course, suppressed.

In summary, we have demonstrated that confinement of $2 \mathrm{~d}$ colloidal crystals by external boundaries has subtle effects on their structure. The least disturbance is caused by 'structured boundaries' (rows frozen in the perfect crystalline order). For planar boundaries, however, the positional LRO is destroyed, even for very thick strips. This phenomenon is not a standard surface-induced melting, however, since a very strongly layered structure (reminiscent of a smectic) is maintained. Just as the $2 \mathrm{~d} X Y$ model below $T_{\mathrm{c}}$ is in a critical state (power-law decay of correlations implies infinite correlation length), the $2 \mathrm{~d}$ crystal is in a similar critical state too, and therefore sensitive to boundary conditions over very large distances. We believe that these effects should be easily observable in experiments involving confined colloids.

\section{Strain-strain correlation functions in a colloidal crystal}

The knowledge of the elastic properties of these model systems is important in the search for novel materials in soft matter physics. Strain-strain correlation functions give access to more detailed information on the elastic behaviour of the system, as they describe the non-local mechanical response of a soft solid in two dimensions. Such non-local elastic effects are important for situations where strain gradients are large [54] or set-ups for which the relevant linear length scales of the solid are of the order of the coarse graining length scale of classical elasticity theory. Both of these criteria are satisfied in colloidal solids under usual experimental conditions [55]. We analyse the fluctuations of particle coordinates in order to gain knowledge of the elastic properties of the colloidal crystal. Recently there has been a lot of interest in obtaining the elastic moduli of soft solids [56, 57] from fluctuations of particle coordinates [51, 46, 58-60]. These methods have several advantages. Particle configurations of the colloidal crystal can be obtained by standard video microscopy [57] and for the calculation of the elastic moduli no external forces need to be applied, which may change the very properties that are being measured.

The description of a $2 \mathrm{~d}$ crystal via a formulation of the elastic free energy as a functional of the strains opens up a way to derive analytic expressions for the strain-strain correlation functions $G_{i j}\left(\vec{x}, \overrightarrow{x^{\prime}}\right)$ in the crystal. These are connected to the non-local compliance $\chi_{i j}$, which is defined as the strain $\varepsilon_{i j}\left(\overrightarrow{x^{\prime}}\right)$ produced at position $\overrightarrow{x^{\prime}}$ due to a stress $\sigma_{i j}(\vec{x})$ at position $\vec{x}$ by $\chi_{i j}=\left(k_{\mathrm{B}} T\right)^{-1} G_{i j}$. The starting point is the dimensionless elastic free energy functional

$$
\mathcal{F}=\begin{array}{cc}
k_{\mathrm{B}} T & 1 \\
a^{2} & 2
\end{array} \int \mathrm{d} \mathbf{r} \sum_{i=1}^{3}\left\{a_{i} e_{i}^{2}+c_{i}\left(\nabla e_{i}\right)^{2}+c_{i}^{\prime}\left(\nabla^{2} e_{i}\right)^{2}\right\} .
$$

Energy and length scales are set by $k_{\mathrm{B}} T$ and the lattice parameter of the triangular lattice $a=(2 /(3 \sqrt{ } \varrho))^{1 / 2}$, respectively. The coefficients $a_{i}(i=1-3)$ are the elastic constants and $c_{i}$, and $c_{i}^{\prime}$ set the length scales over which strain fluctuations decay. The functional is written in a harmonic form of those linear combinations of the strains, which are relevant for a solid in two dimensions: $e_{1}=\partial u_{x} / \partial x+$ $\partial u_{y} / \partial y$ (volume), $e_{2}=\partial u_{x} / \partial x-\partial u_{y} / \partial y$ (deviatoric) and $e_{3}=\left(\partial u_{x} / \partial y+\partial u_{y} / \partial x\right) / 2$ (shear). Care is taken that the colloidal crystal reaches a thermodynamic equilibrium before the measurement. Therefore we can assume $\partial \sigma_{i j} / \partial x_{j}=0$, which ensures the mechanical stability of the crystal. Within classical, linear elasticity theory the strains must fulfil St. Venant's compatibility condition $\nabla \times(\nabla \times \epsilon)^{T}=0$, if there is to be a unique relation between the displacement field and the corresponding strains. Under these constraints only one strain variable is independent and the elastic free 


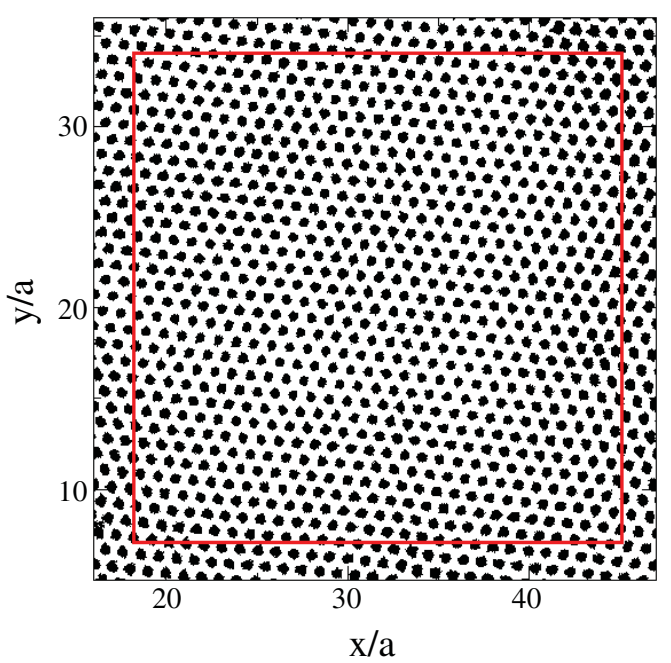

Figure 7. Overlay of all configurations obtained in the crystalline phase at $\Gamma=150$. The boxed section corresponds to the analysed area. The reference lattice was obtained from the average of these particle positions.

energy functional can be written in terms of this strain variable in a harmonic form. Switching to a formulation in Fourier space, the equipartition theorem can be used to extract the analytic prediction for the corresponding strainstrain correlation function. For a more detailed presentation of the derivation see [61]. As an example we present a study of the shear fluctuations in a colloidal crystal. The analysed volume $V$ is embedded in a larger colloidal crystal; therefore these fluctuations can be studied by evaluating the fluctuations in $2 \theta=\left(\partial u_{y} / \partial x-\partial u_{x} / \partial y\right)$. The analytic prediction for the strain-strain correlation function corresponding to this strain variable is given by

$$
\begin{aligned}
& \tilde{G}_{2 \theta} 2 \theta(\mathbf{k} \neq 0)^{-1}=\left(a_{3}+c_{3} k^{2}+c_{3}^{\prime} k^{4}\right. \\
& \left.\quad+\sum_{j=1}^{2}\left(a_{j}+c_{j} k^{2}+c_{j}^{\prime} k^{4}\right)\left(\tilde{\mathcal{Q}}_{j 3}(\mathbf{k})\right)^{2}\right)\left(\tilde{\mathcal{Q}}_{3 \theta}(\mathbf{k})\right)^{2} ; \\
& \tilde{G}_{2 \theta 2 \theta}(0)^{-1}=a_{3} / 4 .
\end{aligned}
$$

Coupled strain variables are related to the independent strain variable via kernels $\tilde{Q}_{i j}: \tilde{e}_{i}=\tilde{Q}_{i j} \tilde{e}_{j}$. The kernels relevant for the analytic formulation of $\tilde{G}_{2 \theta} 2 \theta$ are

$$
\begin{gathered}
\tilde{Q}_{32 \theta}=\frac{1}{2}\left(k_{x}^{4}-k_{y}^{4}\right) /\left(\left(k_{x}^{2}-k_{y}^{2}\right)^{2}+k_{x}^{2} k_{y}^{2}\left(\begin{array}{c}
4 a_{1}+2 a_{3} \\
a_{1}+a_{2}
\end{array}\right)\right) \\
\tilde{\mathcal{Q}}_{13}=\left(4 a_{2}-2 a_{3}\right) /\left(a_{1}+a_{2}\right)\left(k_{x} k_{y}\right) /\left(k^{2}\right) \\
\tilde{\mathcal{Q}}_{23}=-\left(4 a_{1}+2 a_{3}\right) /\left(a_{1}+a_{2}\right)\left(k_{x} k_{y}\right) /\left(k_{x}^{2}-k_{y}^{2}\right) .
\end{gathered}
$$

Experimental data was obtained with an experimental set-up, which is an improved version of [46]. Spherical colloids (diameter $d=4.5 \mu \mathrm{m}$ ) are confined by gravity to a water/air interface formed by a hanging water droplet. The field of view was $835 \times 620 \mu \mathrm{m}^{2}$ containing typically $2 \times 10^{3}$ colloids, whereas the whole system has a size of $50 \mathrm{~mm}^{2}$ and contains about $3 \times 10^{5}$ colloids. The analysed volume $V$ contains 840 particles arranged in a triangular lattice. The particles
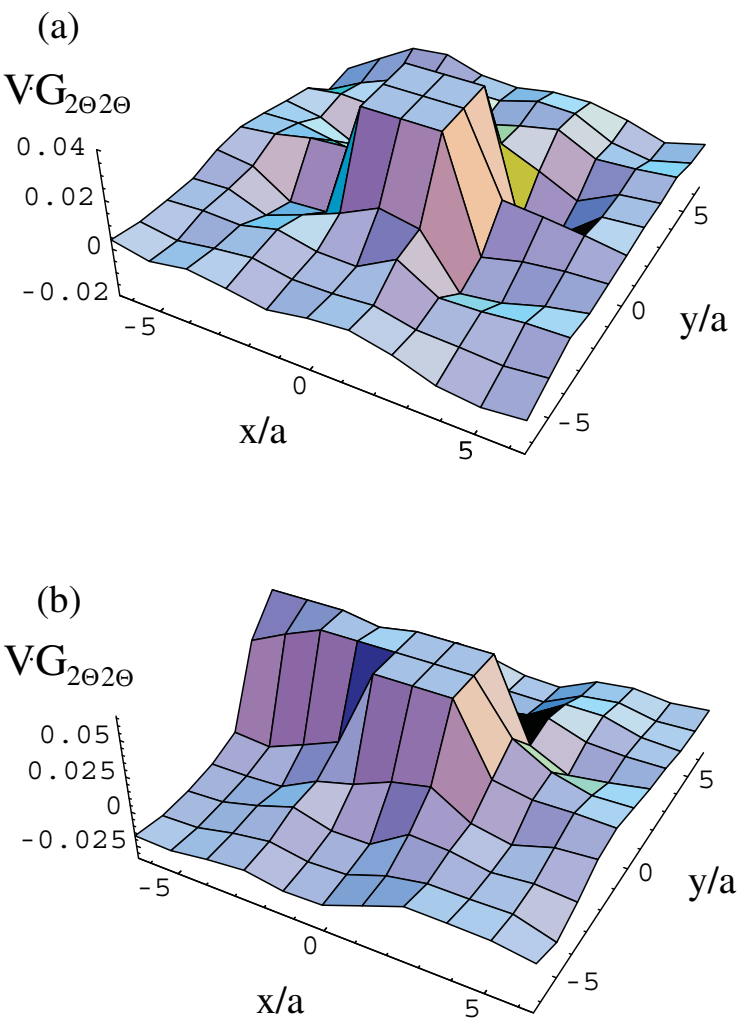

Figure 8. (a) Real space surface plot of the measured strain-strain correlation function $V G_{2 \theta 2 \theta}$. (c) Real space surface plot of $V G_{2 \theta 2 \theta}$ in a sample section containing defects. In both cases the analysed volume is $V=(L / a)^{2}$ with $L=12.881 a$.

are super-paramagnetic. Therefore a magnetic field $\vec{H}$ applied perpendicular to the water/air interface induces a magnetic moment $\vec{M}=\chi_{M} \vec{H}$ in each particle. This leads to a repulsive (inverse cubic) dipole-dipole pair interaction with the dimensionless interaction strength given by $\Gamma$, the ratio of potential to thermal energy. Thus $\Gamma$ can be interpreted as an inverse temperature. Coordinates of all particles at each time step are analysed and the trajectories are recorded for 2-3 h. The data was taken in the solid phase, as can be seen in figure 7 , where an overlay of all configurations obtained during the measurement shows a triangular lattice structure. The boxed section is the analysed volume $V$. Strains are calculated with respect to the average particle positions, as reference (corrected for centre-of-mass motion and rotations of the system) using the scheme of Falk and Langer [62]. Figure 8(a) shows the measured strain-strain correlation function $G_{2 \theta 2 \theta}$ in real space. Cuts in $k$ space along the $k_{x}$ or $k_{y}$ direction of $\tilde{G}_{2 \theta 2 \theta}$ are fitted in order to obtain the shear modulus $\mu=58.6_{a^{2}}^{k_{\mathrm{B}}}$. A different approach [46] to measure the shear modulus of the colloidal crystal is to obtain the probability distribution of the strain variable $2 \theta$. An example of $P(2 \theta)$ measured over boxes of volume $V_{B}=16.0 a^{2}$ is shown in figure $9(\mathrm{~b})$. The mean-square fluctuations in $2 \theta$ are obtained by fitting a normal distribution to the data and extracting its standard deviation. They are related to the shear modulus of the solid via $\mu=\left(V\left\langle(2 \theta)^{2}\right\rangle\right)^{-1}$. The shear modulus obtained according 


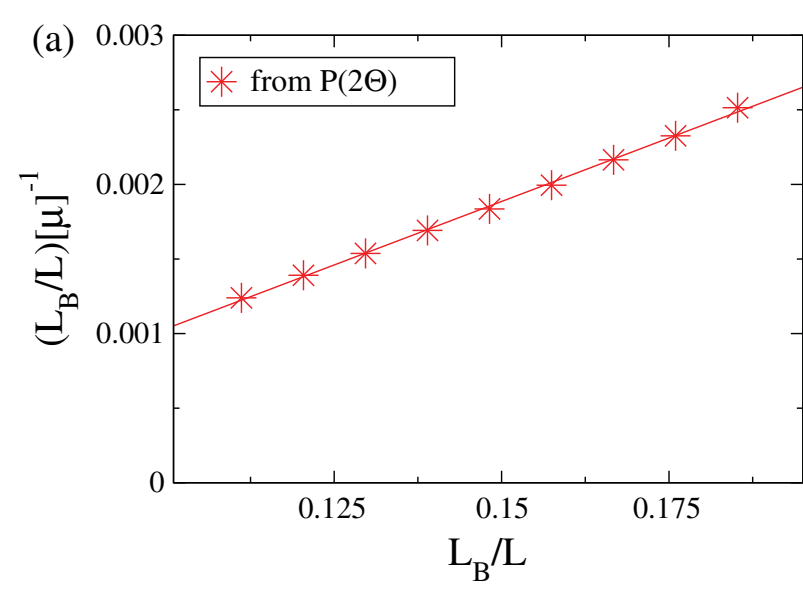

(b)

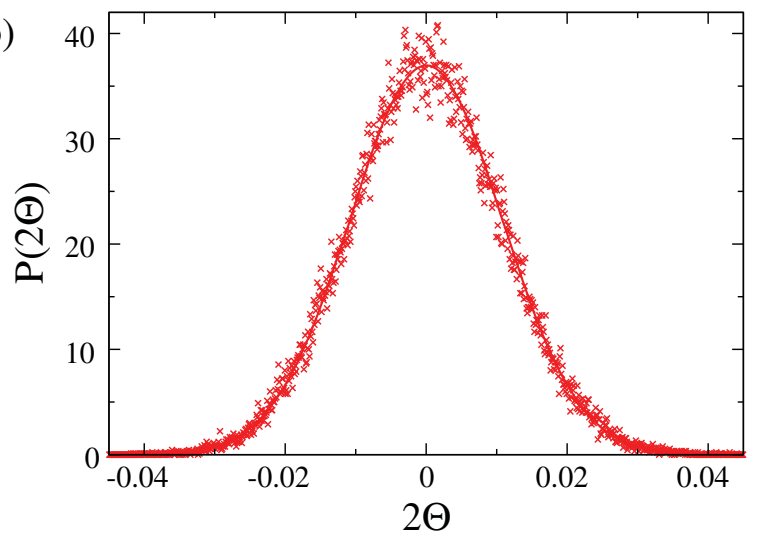

Figure 9. (a) Finite size scaling of the shear modulus obtained from the mean-square fluctuations of $2 \theta$. (b) Example of the measured probability distributions of the fluctuations in the strain variable $2 \theta$ for a sub-volume $V_{B}=16.0 a^{2}$.

to this method is dependent on the size of the analysed subvolume $V_{B}=L_{B}^{2}$. A finite size scaling is used to extract the shear modulus in the thermodynamic limit, which is $\mu=$ $58.7 \frac{k_{\mathrm{B} T}}{a^{2}}$. The finite size scaling is shown in figure 9(a), where the reciprocal value of the shear modulus times the ratio of sub-volume to analysed volume $L_{B} / L$ is plotted as a function of this ratio. From these analysis one can see that the treatment via strain-strain correlation function gives results consistent with established procedures to within less than $1 \%$.

The strain-strain correlation function $\tilde{G}_{2 \theta 2 \theta}(L / a)^{2}$ can furthermore be used to visualize the presence of defects in the crystalline structures. Its spatial symmetry is sensitive to the presence of defects. They lead to a pronounced asymmetry in the correlation function, as displayed in figure 8(b). Here data from a region of the sample containing defects was analysed. As in our analysis displacements are calculated from the average particle positions the strain-strain correlation function shown in figure 8 (b) does not include the singular (Burger's vector) contribution to the displacement from dislocationantidislocation pairs, as the chosen reference lattice already includes the defect pairs themselves.

In summary we have shown that the strain-strain correlation function for shear fluctuations $(T>0)$ in a $2 \mathrm{~d}$ soft solid can be analytically predicted and measured in experiments on colloidal dispersions, thus giving access to the non-local elastic response of colloidal crystals. Elastic moduli extracted by fitting the correlation function along specific directions in Fourier space are consistent with those obtained by other methods. We believe that the strain correlation function may serve as a benchmark for characterizing soft crystals as it is sensitive to the presence of defect pairs.

\section{Acknowledgments}

This work was funded by the Deutsche Forschungsgesellschaft (SFB TR6/C4). Granting of computer time from HLRS, NIC and SSP is gratefully acknowledged.

\section{References}

[1] Chowdhury A, Ackerson B J and Clark N A 1985 Phys. Rev. Lett. 55833

[2] Wei Q-H, Bechinger C, Rudhardt D and Leiderer P 1998 Phys. Rev. Lett. 812606

[3] Chakrabarti J, Krishnamurthy H R, Sood A K and Sengupta S 1995 Phys. Rev. Lett. 752232

[4] Strepp W, Sengupta S and Nielaba P 2001 Phys. Rev. E 63046106

[5] Strepp W, Sengupta S and Nielaba P 2002 Phys. Rev. E 66056109

[6] Strepp W, Sengupta S, Lohrer M and Nielaba P 2002 Comput. Phys. Commun. 147370

Strepp W, Sengupta S, Lohrer M and Nielaba P 2003 Math. Comput. Simul. 62519

[7] Chaudhuri P, Das C, Dasgupta C, Krishnamurthy H R and Sood A K 2005 Phys. Rev. E 72061404

[8] Bürzle F and Nielaba P 2007 Phys. Rev. E 76051112

[9] Chakrabarti J, Krishnamurthy H R and Sood A K 1994 Phys. Rev. Lett. 732923

[10] Frey E, Nelson D R and Radzihovsky L 1999 Phys. Rev. Lett. 832977

[11] Radzihovsky L, Frey E and Nelson D R 2001 Phys. Rev. E 63031503

[12] Franzrahe K and Nielaba P 2007 Phys. Rev. E 76061503

[13] Franzrahe K et al 2005 Comput. Phys. Commun. 169197

[14] Franzrahe K et al 2007 High Performance Computing in Science and Engineering'07 ed W E Nagel, D Kröner and M Resch (Berlin: Springer) p 83

[15] Likos C N and Henley C L 1993 Phil. Mag. B 6885

[16] Lue L and Woodcock L V 1999 Mol. Phys. 961435

[17] Baumgartl J, Dullens R P A, Dijkstra M, Roth R and Bechinger C 2007 Phys. Rev. Lett. 98198303

[18] Hoffmann N, Ebert F, Likos C N, Löwen H and Maret G 2006 Phys. Rev. Lett. 97078303

[19] Gordon C, Dijkstra M, Evans R and Roth R 2005 Mol. Phys. 1033009

[20] Buhot A and Krauth W 1999 Phys. Rev. E 592939

[21] Brader J M et al 2001 Phys. Rev. E 63041405

Schmidt M and Löwen H 1997 Phys. Rev. E 557228 and references therein

[22] Ricci A, Nielaba P, Sengupta S and Binder K 2006 Phys. Rev. E 74 010404(R)

[23] Ricci A, Nielaba P, Sengupta S and Binder K 2007 Phys. Rev. E 75011405

[24] Riste T (ed) 1980 Ordering in Strongly Fluctuating Condensed Matter Systems (New York: Plenum)

[25] Nelson D R 1986 Phase Transitions and Critical Phenomena vol 7 ed C Domb and J L Lebowitz (New York: Academic)

Binder K 1986 Phase Transitions and Critical Phenomena vol 8, ed C Domb and J L Lebowitz (New York: Academic) p 1

Diehl H W 1986 Phase Transitions and Critical Phenomena vol 10, ed C Domb and J L Lebowitz (New York: Academic) p 75 
Cardy J L 1986 Phase Transitions and Critical Phenomena vol 11, ed C Domb and J L Lebowitz (New York: Academic) p 55

[26] Höhler G and Niekisch A (ed) 1992 Critical Phenomena at Surfaces and Interfaces (Springer Tracts in Modern Physics vol 126) (Berlin: Springer)

[27] Binder K, Sengupta S and Nielaba P 2002 J. Phys.: Condens. Matter 142323

[28] Froltsov V A et al 2003 Phys. Rev. E 68061406

[29] Berche B 2003 J. Phys. A: Math. Gen. 36585

[30] Piacente G et al 2004 Phys. Rev. B 69045324

[31] Haghgooie R and Doyle P S 2004 Phys. Rev. E 70061408

[32] Haghgooie R and Doyle P S 2005 Phys. Rev. E 72011405

[33] Köppl M, Henseler P, Erbe A, Nielaba P and Leiderer P 2006 Phys. Rev. Lett. 97208302

[34] Glasson P et al 2001 Phys. Rev. Lett. 87176802

[35] Lai Y-J and Lin I 2001 Phys. Rev. E $64015601(\mathrm{R})$ Teng L W et al 2003 Phys. Rev. Lett. 90245004

[36] Chaudhuri D and Sengupta S 2004 Phys. Rev. Lett. 93115702

[37] Pieranski P et al 1980 Mol. Phys. 40225

[38] Emery V J and Axe J D 1978 Phys. Rev. Lett. 401507

[39] Axe J D 1980 Ordering in Strongly Fluctuating Condensed Matter Systems (New York: Plenum) p 399

[40] Binder K 1995 Cohesion and Structure at Surfaces ed D G Pettifor (Amsterdam: Elsevier) p 121

[41] Poon W C and Pusey P N 1995 Observation, Prediction and Simulation of Phase Transitions in Complex Fluids ed M Baus et al (Dordrecht: Kluwer) p 3

Lekkerkerker H N W et al 1995 Observation, Prediction and Simulation of Phase Transitions in Complex Fluids ed M Baus et al (Dordrecht: Kluwer) p 53

[42] Palberg T 1997 Curr. Opin. Colloids Interface Sci. 2607

[43] Löwen H 2001 J. Phys.: Condens. Matter 13 R415

[44] Löwen H and Likos C N (ed) 2004 Colloidal dispersions in external fields J. Phys.: Condens. Matter 16 (whole special issue, number 38 )

[45] Zahn K et al 1999 Phys. Rev. Lett. 822781
Zahn K and Maret G 2000 Phys. Rev. Lett. 853656

[46] Zahn K, Wille A, Maret G, Sengupta S and Nielaba P 2003 Phys. Rev. Lett. 90155506

[47] Bagchi K, Andersen H C and Swope W 1996 Phys. Rev. E 539794

[48] Landau D P and Binder K 2005 A Guide to Monte Carlo Simulations in Statistical Physics 2nd edn (Cambridge: Cambridge University Press)

[49] Mermin N D 1968 Phys. Rev. 176250

[50] Ostlund S and Halperin B I 1981 Phys. Rev. B 23335

[51] Sengupta S, Nielaba P, Rao M and Binder K 2000 Phys. Rev. E 611072

Sengupta S, Nielaba P and Binder K 2000 Phys. Rev. E 616294

[52] Nielaba P, Binder K, Chaudhuri D, Franzrahe K, Henseler P, Lohrer M, Ricci A, Sengupta S and Strepp W 2004 J. Phys.: Condens. Matter 16 S4115

[53] Müller P and Saul A 2004 Surf. Sci. Rep. 54157

[54] Eringen A C 2002 Nonlocal Continuum Field Theories (Heidelberg: Springer)

[55] Witten T A 2005 J. Phys.: Condens. Matter 17 S1651

[56] Daoud M and Williams C E (ed) 1999 Soft Matter Physics (Heidelberg: Springer)

[57] Pusey P N 2005 Science E 3091198

Ramirez-Saito A, Bechinger C and Arauz-Lara J L 2006 Phys. Rev. E 74 030401(R)

[58] Keim P, Maret G, Herz U and von Grünberg H-H 2004 Phys. Rev. Lett. 92215504

[59] von Grünberg H-H, Keim P, Zahn K and Maret G 2004 Phys. Rev. Lett. 93255703

Zanghellini J, Keim P and von Grünberg H-H 2005 J. Phys.: Condens. Matter 173579

[60] Reinke D, Stark H, von Grünberg H-H, Schofield A B, Maret G and Gasser U 2007 Phys. Rev. Lett. 98038301

[61] Franzrahe K, Keim P, Maret G, Nielaba P and Sengupta S 2008 Phys. Rev. E in press

[62] Falk M L and Langer J S 1998 Phys. Rev. E 577192 